\title{
SARS-CoV-2 testing in patients with low COVID-19 suspicion at admission to a tertiary care hospital, Stockholm, Sweden, March to September 2020
}

\author{
Ana Requena-Méndez 1,2,3 , Aikaterini Mougkou4 ${ }^{4}$, Pontus Hedberg ${ }^{1,2}$, Suzanne D van der Werff', , Hideyuki Tanushi' ${ }^{1,5}$, Olof \\ Hertting ${ }^{4}$, Anna Färnert ${ }^{1,2}$, Filippa Nyberg ${ }^{6}$, Pontus Naucler ${ }^{1,2}$, COVID-19-data-review collaborators ${ }^{7}$ \\ 1. Division of Infectious Diseases, Department of Medicine Solna, Karolinska Institutet, Solna, Sweden \\ 2. Department of Infectious Diseases, Karolinska University Hospital, Solna, Sweden \\ 3. Barcelona Institute for Global Health, ISGlobal, Hospital Clinic-Universitat de Barcelona, Barcelona, Spain \\ 4. Department of Pediatrics, Karolinska University Hospital, Solna, Sweden \\ 5. Department of Data Processing and Analysis, Karolinska University Hospital, Solna, Sweden \\ 6. Division of Dermatology and Venereology, Department of Medicine Solna, Karolinska Institutet, Solna Sweden \\ 7. The COVID-19-data-review collaborators are listed under Investigators
}

Correspondence: Ana Requena-Méndez (requena.mendez@ki.se)

Investigators: The investigators are listed at the end of the article.

Citation style for this article:

Requena-Méndez Ana, Mougkou Aikaterini, Hedberg Pontus, van der Werff Suzanne D, Tanushi Hideyuki, Hertting Olof, Färnert Anna, Nyberg Filippa, Naucler Pontus, COVID-19-data-review collaborators. SARS-CoV-2 testing in patients with low COVID-19 suspicion at admission to a tertiary care hospital, Stockholm, Sweden, March to September 2020. Euro Surveill. 2022;27(7):pii=2100079. https://doi.org/10.2807/1560-7917.ES.2022.27.7.2100079

Background: Universal SARS-CoV-2 testing at hospital admission has been proposed to prevent nosocomial transmission. Aim: To investigate SARS-CoV-2 positivity in patients tested with low clinical COVID-19 suspicion at hospital admission. Methods: We characterised a retrospective cohort of patients admitted to Karolinska University Hospital tested for SARS-CoV-2 by PCR from March to September 2020, supplemented with an in-depth chart review (16 March-12 April). We compared positivity rates in patients with and without clinical COVID-19 suspicion with Spearman's rank correlation coefficient. We used multivariable logistic regression to identify factors associated with test positivity. Results: From March to September 2020, $66.9 \%(24,245 / 36,249)$ admitted patient episodes were tested; of those, $61.2 \%(14,830 / 24,245)$ showed no clinical COVID-19 suspicion, and the positivity rate was $3.2 \%(469 / 14,830)$. There was a strong correlation of SARS-CoV-2 positivity in patients with low vs high COVID-19 suspicion (rho=0.92; $\mathrm{p}<0.001$ ). From 16 March to 12 April, the positivity rate was $3.9 \%$ $(58 / 1,482)$ in individuals with low COVID-19 suspicion, and $3.1 \%(35 / 1,114)$ in asymptomatic patients. Rates were higher in women $(5.0 \%$; 45/893) vs men (2.0\%; 12/589; $p=0.003$ ), but not significantly different if pregnant women were excluded $(3.7 \%(21 / 566)$ vs $2.2 \%(12 / 589) ; p=0.09)$. Factors associated with SARS-CoV-2 positivity were testing of pregnant women before delivery (odds ratio (OR): 2.6 ; $95 \%$ confidence interval (CI): $1.3-5.4)$ and isolated symptoms in adults (OR: $3.3 ; 95 \% \mathrm{Cl}: 1.8-6.3$ ). Conclusions: This study shows a relatively high SARS-CoV-2 positivity rate in patients with low COVID-19 suspicion upon hospital admission. Universal SARS-CoV-2 testing of pregnant women before delivery should be considered.

\section{Introduction}

Since the first cases of coronavirus disease 2019 (COVID19) were identified in Wuhan, China, in December 2019, the global pandemic caused by severe acute respiratory syndrome coronavirus-2 (SARS-CoV-2) has led, as at January 2022 , to more than 5,600,000 deaths [1]. Although the World Health Organization advocated widespread SARS-CoV-2 testing [2], national capacities for implementing this strategy have diverged considerably because of inadequate testing capacity [3,4].

Hospital screening at admission was implemented from the first weeks of the pandemic to further limit SARS-CoV-2 spread to inpatients and frontline healthcare workers [5]. Symptom-based testing for COVID19 has shown to be specific but not sensitive, since absence of clinical symptoms does not rule out infections [5]. Furthermore, during the early pandemic, findings emerged suggesting that the virus may spread from asymptomatic or pre-symptomatic individuals $[6,7]$. SARS-CoV-2 testing approaches focussed solely on the presence of symptoms are therefore unlikely to be adequate to prevent nosocomial spread if there is a sustained community transmission risk [5]. Some hospitals imposed screening to prevent SARS-CoV-2 transmission to operating theatres, oncology units, and labour and delivery wards [8-10], whereas in other hospital units, testing was only performed in symptomatic patients [11]. 
Flowchart of the study population with in-hospital healthcare episodes and SARS-CoV-2 tests, Karolinska University Hospital, Stockholm, Sweden, 16 March-27 September 2020 ( $\mathrm{n}=36,249$ healthcare episodes)

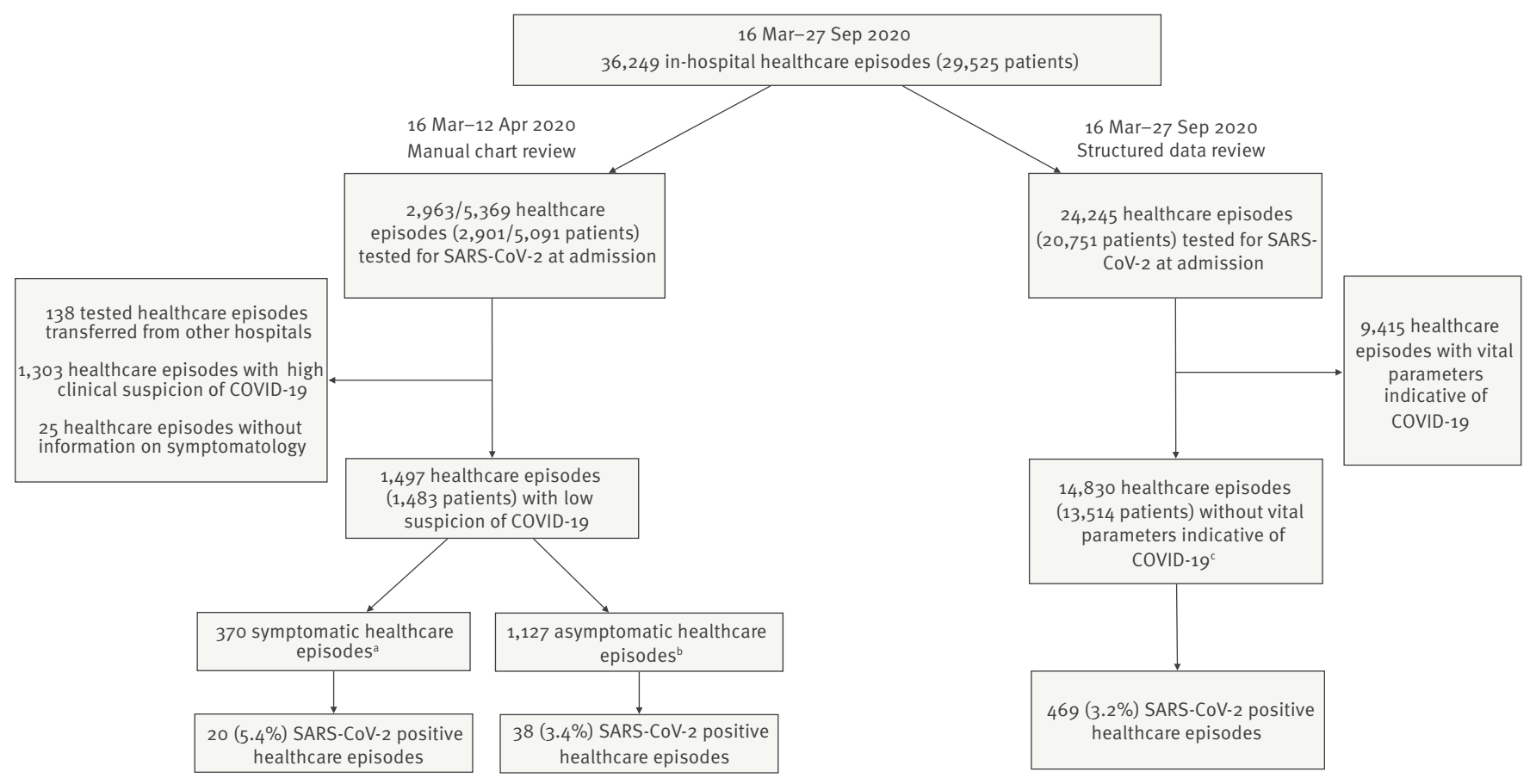

COVID-19: coronavirus disease; SARS-CoV-2: severe acute respiratory syndrome coronavirus 2.

${ }^{a}$ Low clinical suspicion of COVID-19 with isolated symptoms (fever, cough, fatigue, dyspnoea, myalgia, diarrhoea, abdominal pain, vomiting or headache).

${ }^{b}$ Low clinical suspicion of COVID-19 without fever, cough, fatigue, dyspnoea, myalgia, diarrhoea, abdominal pain, vomiting and headache.

${ }^{\mathrm{C}}$ Health care episodes with body temperature $<38^{\circ} \mathrm{C}$, saturation $>95 \%$ and age-dependent increased respiratory rate ( $\leq 60$ when $<12$ months, $\leq 40$ when $1-3$ years, $\leq 34$ when $4-5$ years, $\leq 30$ when $6-12$ years, $\leq 20$ when $>12$ years).

A report published in 2020 by the European Centre for Disease Prevention and Control (ECDC) on screening strategies recommends that all patients should be tested for SARS-CoV-2 on admission [12]. Whether the use of universal screening on admission is an efficient way of resource allocation to minimise the risk of nosocomial transmission of the virus is depending on the circulation of newly infected individuals in the community what differ among areas and over time [13].

By 15 January 2022, 2,015,276 (19.5\%) individuals in the Swedish population have tested positive for SARSCoV-2 and $0.8 \%$ of these died [14]. Stockholm has been one of the most affected regions, with 472,169 (20.1\%) confirmed COVID-19 cases and 4,700 deaths (1.0\% of positive cases), although only hospital admissions were tested for SARS-CoV-2 in the spring of 2020 , resulting in an underestimated number of cases and an overestimated mortality rate [15]. From 25 March 2020 onward, several clinics at Karolinska University Hospital in Stockholm recommended routine SARSCoV-2 testing to patients admitted to the hospital. This included testing before certain procedures, e.g. before delivery, immunosuppressive treatment, surgery, and other invasive procedures such as endoscopy or bronchoscopy as well as all paediatric admissions (until September 2020), irrespective of whether patients were having a low or high COVID-19 clinical suspicion.

The primary aim of this study was to assess the proportion of positive SARS-CoV-2 cases identified during healthcare episodes with a low clinical suspicion of COVID-19 tested at hospital admission to Karolinska University Hospital, and to further investigate factors associated with SARS-CoV-2-positive test results among these patients. The secondary aim was to investigate the value of SARS-CoV-2 testing in relation to the background hospitalisation rate because of COVID-19 in Region Stockholm.

\section{Methods}

\section{Study design and setting}

We conducted a retrospective cohort study of all admitted patients, including adults and children, tested for SARS-CoV-2 from 16 March to 27 September 2020 at Karolinska University Hospital (which has two locations in Huddinge and Solna), Stockholm, Sweden. This is a 
SARS-CoV-2 positivity rate in patients with low clinical suspicion of COVID-19, Karolinska University Hospital, Stockholm, Sweden

A. SARS-CoV-2 testing, positivity rate and COVID-19 hospitalisations in patients without fever and dyspnoea ${ }^{a}$

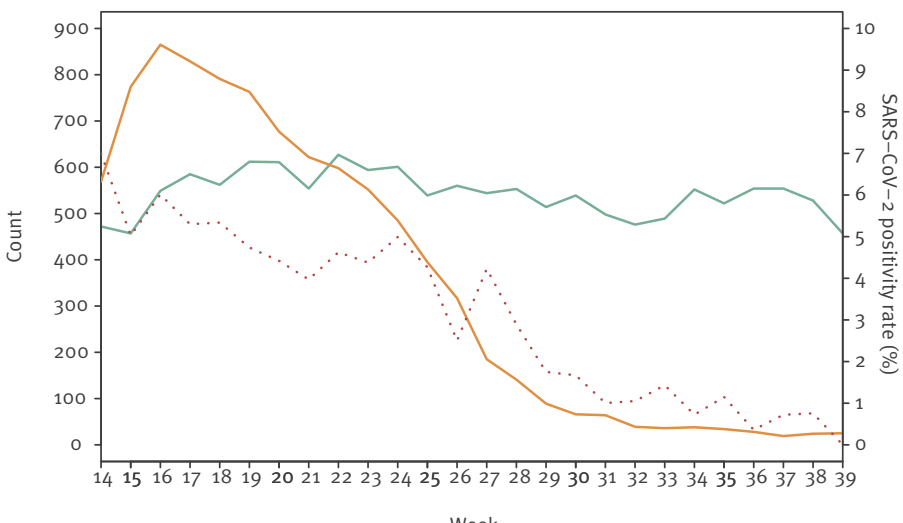

Week

- Number of COVID-19 hospitalisations in Region Stockholm (0-900)

- SARS-CoV-2 tested at Karolinska University Hospital without fever and dyspnoea (0-90o)

... SARS-CoV-2 positivity rate at Karolinska University Hospital without fever and dyspnoea (0-10)

\section{Correlation between hospitalised patients with $^{\mathrm{b}}$ or without ${ }^{\mathrm{a}}$} fever and dyspnoea

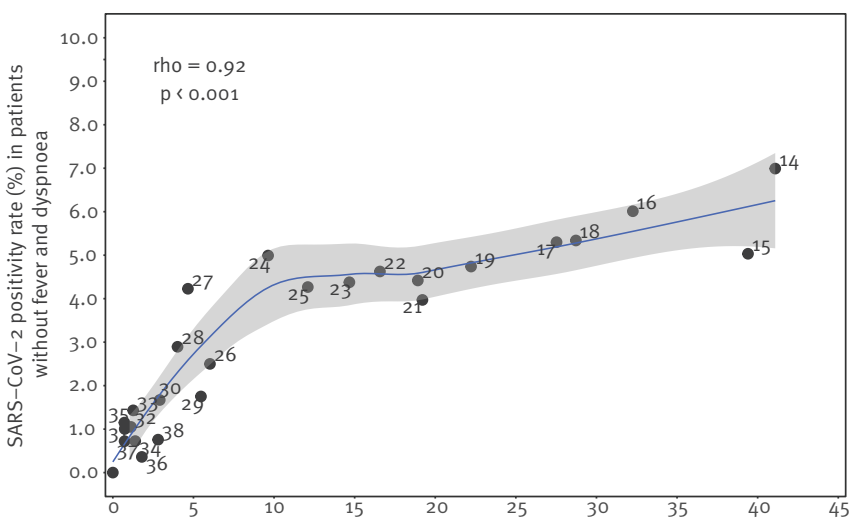

Positivity rate (\%) in patients with fever or dyspnoea
B. Correlation between SARS-CoV-2 positivity rate in patients without fever and dyspnoea ${ }^{a}$ and COVID-19 hospitalisation rate

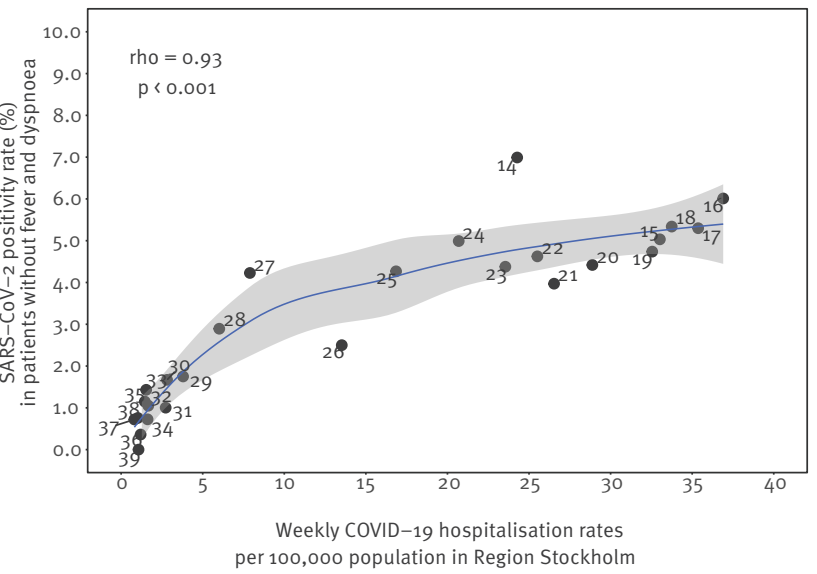

COVID-19: coronavirus disease; KUH: Karolinska University Hospital; SARS-CoV-2: severe acute respiratory syndrome coronavirus 2.

a Presenting on admission with a body temperature of $<38^{\circ} \mathrm{C}$, AND an oxygen saturation of $>95 \%$, AND a respiratory rate depending on age as follows: $<60$ for $<12$ months, $<40$ for $1-3$ years, $<34$ for $4-5$ years, $<30$ for $6-12$ years, and $<20$ for $>12$ years on admission.

${ }^{b}$ Presenting on admission with an increased body temperature OR decreased oxygen saturation OR increased respiratory rate according to footnote a.

Comparisons of SARS-CoV-2 positivity rate in patients without vital parameters indicative of COVID-19 at Karolinska University Hospital and Region Stockholm COVID-19 hospitalisations and hospitalisation rates per 100,000 population by week. Panel A shows the number of SARS-CoV-2 tests (green line) and the corresponding positivity rate (red dashed line) in patients without fever and dyspnoea at Karolinska University Hospital in relation to the number of COVID-19 hospitalisations in Region Stockholm (orange line). Panel B shows the correlation between the weekly Region Stockholm COVID-19 hospitalisation rate per 100,000 population (black solid circles represent week numbers in 2020) and the weekly positivity rate in patients without fever and dyspnoea at Karolinska University Hospital. Panel C shows the correlation between the weekly positivity rate in patients with (x-axis) or without (y-axis) fever and dyspnoea.

For Panels B and C, rho represents the Spearman's rank correlation coefficient. The blue line represents a fitted general additive model and the grey area represents the $95 \%$ confidence interval of the model. 
tertiary care academic hospital with 1,100 beds divided between two sites, which serves a population of 2.3 million inhabitants, i.e. the entire population of Region Stockholm. The hospital in Solna is a modern facility that opened in 2017 where most beds are set in private rooms, whereas the hospital in Huddinge includes beds that are located in shared rooms.

\section{Data sources}

We used two sources of healthcare data for this study. First, we used a pseudo-anonymised research database that contains demographic, clinical and microbiological information on all inpatients at Karolinska University Hospital to examine those tested for SARSCoV-2 at admission from 16 March to 27 September 2020. The research dataset from the entire study period only included structured variables.

Second, to have more information on the reasons for testing and clinical symptoms, we performed an indepth manual medical chart review of all admitted patients who received a SARS-CoV-2 test during the peak of virus transmission between 16 March to 12 April 2020, using the hospital electronic health record system, as part of a quality assurance project. For the in-depth medical chart review, medical records of healthcare episodes with at least one PCR test for SARS-CoV-2 were manually reviewed; we registered (i) reasons for testing as noted by health professionals, (ii) demographic characteristics, (iii) information on medical history and (iv) clinical and laboratory data when available. All patients in the chart review are included in the research database; however, because personal identification numbers were not available in the research database, it was not possible to directly link the two data sources.

\section{SARS-CoV-2 testing}

From 1 March 2020, all patients with COVID-19-related symptoms were recommended to be tested for SARSCoV-2 infection. From 25 March 2020 onward, several clinics at Karolinska University Hospital recommended routine SARS-CoV-2 testing to patients admitted to the hospital (before delivery, immunosuppressive treatment, surgery, and other invasive procedures such as endoscopy or bronchoscopy as well as all paediatric admissions), restricted to tests performed from $24 \mathrm{~h}$ before until $48 \mathrm{~h}$ after hospital admission. Children were considered those aged $<18$ years. For both datasets, PCR tests were performed at the Karolinska University Laboratory according to routine quality assured procedures for SARS-CoV-2 RNA detection (Supplementary File S1).

A 'healthcare episode' was defined as the time from patient admission until discharge from the hospital. New hospital admissions occurring within $12 \mathrm{~h}$ following discharge were considered as part of the same healthcare episode.

\section{Case definitions}

The case definition for patients considered to have a high or low clinical suspicion of COVID-19 varied depending on which dataset was used, i.e. the research dataset (entire study period) vs in-depth medical chart review (16 March to 27 September 2020).

For the research data set, patients admitted with fever and dyspnoea were considered to have high clinical suspicion of COVID-19. Therefore, we considered patients without fever and dyspnoea on admission as a proxy for low COVID-19 suspicion, i.e. temperature $<38^{\circ} \mathrm{C}$ AND oxygen saturation $\geq 95 \%$ AND respiratory rate depending on age $(\leq 60$ breaths/min for $<12$ months, $\leq 40$ for $1-3$ years, $\leq 34$ for $4-5$ years, $\leq 30$ for 6-12 years, and $\leq 20$ for $\geq 12$ years).

For the in-depth manual medical chart review, the reason for testing was defined as "high clinical suspicion of COVID-19' if there were clinically compatible symptoms indicative of SARS-CoV-2 infection including fever AND any respiratory symptoms (cough, dyspnoea, sore throat), and/or if health professionals suspected COVID-19. Healthcare episodes with a high clinical suspicion of COVID-19 together with other high-risk exposure episodes, e.g. transfer from a nursing home stay or from another hospital OR previous hospitalisation, were excluded from further analysis. Testing in healthcare episodes that did not fulfil this definition of high clinical suspicion were regarded as 'screened with low clinical suspicion of COVID-19'. Yet, since symptoms of COVID-19 can be diffuse, we further assessed these patients according to presence of any symptoms that could be indicative of SARS-CoV-2 infection compared with asymptomatic patients. Individuals were considered asymptomatic if they had no COVID-19-related symptoms including fever, cough, sore throat, fatigue, dyspnoea, myalgia, diarrhoea, abdominal pain, vomiting or headache.

Reasons for testing among those with low COVID-19 suspicion were divided into 'before surgery' or any other invasive procedure', 'before delivery' and testing at hospital admission when the reason for admission was 'other than surgery or invasive procedure'.

\section{Data sources and analysis}

The primary outcome was the positivity rate among all individuals with low clinical COVID-19 suspicion. We also examined the positivity rate in asymptomatic patients. Both were evaluated during the 4-week study period beginning on the 16 March 2020. In addition, factors that could be associated with the SARS-CoV-2 infection, i.e. demographics, underlying conditions, symptoms and reasons for testing, were investigated. The testing rate was expressed as the percentage of total SARS-CoV-2 tests conducted per healthcare episode. If there were several healthcare episodes for one patient during the 4 -week period, the first episode tested was selected to calculate the positivity rate. 
TABLE 1

General characteristics and SARS-CoV-2 positivity rate of patients tested with a low clinical suspicion of COVID-19, Karolinska University Hospital, Stockholm, Sweden, March 16-April 12 ( $\mathrm{n}=1,482)$

\begin{tabular}{|c|c|c|c|c|c|c|c|c|c|c|c|c|c|c|}
\hline \multirow{3}{*}{ Characteristics } & \multicolumn{4}{|c|}{$\begin{array}{l}\text { Hospital admission (not } \\
\text { surgical or delivery) } \\
\qquad(\mathrm{n}=831)^{\mathrm{a}}\end{array}$} & \multicolumn{4}{|c|}{$\begin{array}{l}\text { Before surgical or invasive } \\
\text { procedure } \\
\qquad(n=324)\end{array}$} & \multirow{2}{*}{\multicolumn{2}{|c|}{\begin{tabular}{|c}
$\begin{array}{c}\text { Before delivery } \\
(n=327)\end{array}$ \\
Adults \\
$(n=327)$
\end{tabular}}} & \multicolumn{4}{|c|}{$\begin{array}{c}\text { Total } \\
(\mathrm{n}=1,482)^{\mathrm{a}}\end{array}$} \\
\hline & \multicolumn{2}{|c|}{$\begin{array}{l}\text { Adults } \\
(n=647)\end{array}$} & \multicolumn{2}{|c|}{$\begin{array}{l}\text { Children } \\
(n=184)\end{array}$} & \multicolumn{2}{|c|}{$\begin{array}{l}\text { Adults } \\
(n=249)\end{array}$} & \multicolumn{2}{|c|}{$\begin{array}{l}\text { Children } \\
(\mathrm{n}=75)\end{array}$} & & & \multicolumn{2}{|c|}{$\begin{array}{l}\text { Adults } \\
(n=1,223)\end{array}$} & \multicolumn{2}{|c|}{$\begin{array}{l}\text { Children } \\
(n=259)\end{array}$} \\
\hline & $n$ & $\%$ & $\mathrm{n}$ & $\%$ & $\mathrm{n}$ & $\%$ & $\mathrm{n}$ & $\%$ & n & $\%$ & $\mathrm{n}$ & $\%$ & $\mathrm{n}$ & $\%$ \\
\hline \multicolumn{15}{|l|}{ Sex } \\
\hline Female & 336 & 51.9 & 84 & 45.7 & 121 & 48.6 & 25 & 33.3 & 327 & 100 & 784 & 64.1 & 109 & 42.1 \\
\hline Male & 311 & 48.1 & 100 & 54.3 & 128 & 51.4 & 50 & 66.7 & 0 & 0 & 439 & 35.9 & 150 & 57.9 \\
\hline \multicolumn{15}{|l|}{ Age (years) } \\
\hline Median (IQR) ${ }^{\mathrm{a}}$ & \multicolumn{2}{|c|}{$\begin{array}{c}59.0 \\
(42.3-71.3)\end{array}$} & \multicolumn{2}{|c|}{$\begin{array}{c}4.6 \\
(0.1-11.8) \\
\end{array}$} & \multicolumn{2}{|c|}{$\begin{array}{c}66.4 \\
(45.1-78.5) \\
\end{array}$} & \multicolumn{2}{|c|}{$\begin{array}{c}6.7 \\
(2.6-12.3) \\
\end{array}$} & \multicolumn{2}{|c|}{$\begin{array}{c}31.7 \\
(28.1-35.2)\end{array}$} & \multicolumn{2}{|c|}{$\begin{array}{c}49.4 \\
(33.0-68.3) \\
\end{array}$} & \multicolumn{2}{|c|}{$\begin{array}{c}5.6 \\
(0.4-12.3) \\
\end{array}$} \\
\hline \multicolumn{15}{|l|}{ Comorbidities } \\
\hline Hypertension & $221 / 645$ & 34.3 & 0 & 0 & 99 & 39.8 & 0 & 0 & 6 & 1.8 & 326 & 26.7 & o & 0 \\
\hline Cardiovascular diseases & $216 / 645$ & 33.5 & 1 & 0.5 & 93 & 37.3 & 3 & 4.0 & 4 & 1.2 & 316 & 25.6 & 4 & 1.5 \\
\hline Diabetes mellitus & $88 / 645$ & 13.6 & 3 & 1.6 & 25 & 10.0 & 0 & 0 & 4 & 1.2 & 117 & 9.6 & 3 & 1.2 \\
\hline Chronic respiratory diseases & $77 / 646$ & 11.9 & 6 & 3.3 & 21 & 8.4 & 3 & 4.0 & 1 & 0.3 & 99 & 8.1 & 9 & 3.5 \\
\hline Chronic hepatic diseases & $34 / 644$ & 5.3 & 0 & 0 & 11 & 4.4 & 1 & 1.3 & 1 & 0.3 & 46 & 3.8 & 1 & 0.4 \\
\hline Chronic renal diseases & $59 / 643$ & 9.2 & 5 & 2.7 & 23 & 9.2 & 3 & 4.0 & 0 & 0 & 82 & 6.7 & 8 & 3.1 \\
\hline Cancer & $184 / 645$ & 28.5 & $17 / 182$ & 9.3 & 79 & 31.7 & 8 & 10.7 & 0 & 0 & 263 & 21.5 & 25 & 9.7 \\
\hline Transplant & $34 / 644$ & 5.3 & 1 & 0.5 & 3 & 1.2 & $3 / 74$ & 4.1 & 1 & 0.3 & 38 & 3.1 & 5 & 1.9 \\
\hline Autoimmune diseases & $46 / 645$ & 7.1 & 1 & 0.5 & $24 / 247$ & 9.7 & $1 / 74$ & 1.4 & 1 & 0.3 & 71 & 5.8 & 2 & 0.8 \\
\hline Current immunosuppression & $89 / 645$ & 13.8 & 10 & 5.4 & 18 & 7.2 & $11 / 74$ & 14.9 & 2 & 0.6 & 109 & 8.9 & 21 & 8.1 \\
\hline Neuromuscular disorders & $13 / 644$ & 2.0 & 3 & 1.6 & 6 & 2.4 & 1 & 1.3 & 0 & 0 & 19 & 1.6 & 4 & 1.5 \\
\hline \multicolumn{15}{|l|}{ SARS-CoV-2 infection } \\
\hline $\begin{array}{l}\text { Patients with low COVID-19 } \\
\text { clinical suspicion }\end{array}$ & $20 / 647$ & 3.1 & $5 / 184$ & 2.7 & $6 / 249$ & 2.4 & $2 / 75$ & 2.7 & $24 / 327$ & $7 \cdot 3$ & $50 / 1,223$ & 4.1 & $7 / 259$ & 2.7 \\
\hline $\begin{array}{l}\text { Asymptomatic COVID-19 } \\
\text { patients }\end{array}$ & $8 / 413$ & 1.9 & $2 / 104$ & 1.9 & $3 / 206$ & 1.5 & $2 / 69$ & 2.9 & $20 / 322$ & 6.2 & $31 / 941$ & 3.3 & $4 / 173$ & 2.3 \\
\hline \multicolumn{15}{|c|}{ Municipality by socioeconomic index ${ }^{b}$} \\
\hline Level & \multicolumn{2}{|c|}{$\mathrm{n}=520$} & \multicolumn{2}{|c|}{$\mathrm{n}=153$} & \multicolumn{2}{|c|}{$\mathrm{n}=192$} & \multicolumn{2}{|c|}{$\mathrm{n}=53$} & \multicolumn{2}{|c|}{$\mathrm{n}=296$} & $\mathrm{n}=1,0$ & 008 & $\mathrm{n}=2$ & 206 \\
\hline Low & 72 & 13.8 & 19 & 12.4 & 26 & 13.5 & 6 & 11.3 & 37 & 12.5 & 135 & 13.4 & 25 & 12.1 \\
\hline Medium & 174 & 33.5 & 67 & 43.8 & 69 & 35.9 & 24 & $45 \cdot 3$ & 114 & 38.5 & 357 & 35.4 & 91 & 44.2 \\
\hline High & 274 & 52.7 & 67 & 43.8 & 97 & 53.5 & 23 & 43.4 & 145 & 49.0 & 516 & 49.9 & 90 & 43.7 \\
\hline
\end{tabular}

COVID-19: coronavirus disease; IQR: interquartile range; SARS-CoV-2: severe acute respiratory syndrome coronavirus 2. Low clinical

suspicion: COVID-19 cases without clinically compatible symptoms indicative of SARS-CoV-2 infection including fever AND any respiratory symptoms (cough, dyspnoea, sore throat), and if health professionals were not registering the clinical suspicion of COVID-19.

a One individual has missing data on age.

${ }^{b}$ Data on municipality was unknown for 215 individuals.

Number of observations is $\mathrm{n}$ if not otherwise specified. Adults are considered those aged $\geq 18$ years and children as those aged $<18$ years. 
TABLE 2

Characteristics associated with SARS-CoV-2 infection in adult patients with low COVID-19 suspicion, Karolinska University Hospital, Stockholm, Sweden, 16 March-12 April $2020(\mathrm{n}=1,223)$

\begin{tabular}{|c|c|c|c|c|c|c|c|}
\hline \multirow[t]{2}{*}{ Characteristics } & \multicolumn{2}{|c|}{$\begin{array}{l}\text { Positive SARS-CoV-2 test } \\
\qquad(\mathrm{n}=50)^{\mathrm{a}}\end{array}$} & \multicolumn{2}{|c|}{ Negative SARS-CoV-2 test $(n=1,173)^{a}$} & \multirow[t]{2}{*}{ OR } & \multirow[t]{2}{*}{$95 \% \mathrm{Cl}^{\mathrm{b}}$} & \multirow[t]{2}{*}{$\mathrm{p}$ value } \\
\hline & $\mathrm{n}$ & $\%$ & $\mathrm{n}$ & $\%$ & & & \\
\hline \multicolumn{8}{|l|}{ Sex } \\
\hline Female & 42 & 84.0 & 742 & 63.3 & \multirow{2}{*}{0.4} & \multirow{2}{*}{$0.2-0.8$} & \multirow{2}{*}{0.02} \\
\hline Male & 8 & 16.0 & 431 & 36.7 & & & \\
\hline \multicolumn{8}{|l|}{ Age (years) } \\
\hline Median (IQR) & \multicolumn{2}{|c|}{$35.6(32.0-62.8)$} & \multicolumn{2}{|c|}{$50.3(33.1-69.0)$} & 0.99 & $0.098-1.01$ & 0.3 \\
\hline \multicolumn{8}{|l|}{ Comorbidities } \\
\hline Hypertension & 10 & 20.0 & $316 / 1,171$ & 27.0 & 1.1 & $0.5-2.8$ & 0.8 \\
\hline Cardiovascular disorders & 6 & 12.0 & $307 / 1,171$ & 26.2 & 0.5 & $0.2-1.4$ & 0.2 \\
\hline Diabetes mellitus & 5 & 10.0 & $112 / 1,171$ & 9.6 & 1.6 & $0.6-4.4$ & 0.3 \\
\hline Chronic respiratory disorder & 4 & 8.0 & $95 / 1,172$ & 8.1 & 1.3 & $0.4-4.0$ & 0.5 \\
\hline Chronic hepatic disorder & 3 & 6.0 & $43 / 1,170$ & 3.7 & 2.3 & $0.7-8.0$ & 0.2 \\
\hline Chronic renal failure & 1 & 2.0 & $81 / 1,168$ & 6.9 & 0.4 & $0.5-2.9$ & 0.3 \\
\hline Cancer & 4 & 8.0 & $259 / 1,171$ & 22.1 & 0.4 & $0.1-1.2$ & 0.09 \\
\hline Autoimmune disease & $5 / 49$ & 10.2 & $66 / 1,170$ & 5.6 & 2.2 & $0.8-5.8$ & 0.1 \\
\hline Immunosuppression & 2 & 4.0 & $107 / 1,171$ & 9.1 & 0.5 & $0.1-2.3$ & 0.4 \\
\hline Transplantation & 1 & 2.0 & $37 / 1,170$ & 3.2 & 0.9 & $0.1-7.2$ & 1.0 \\
\hline \multicolumn{8}{|l|}{ Symptoms } \\
\hline Fever (without respiratory symptoms) & 5 & 10.0 & 60 & 5.1 & 2.8 & $1.1-7.6$ & 0.039 \\
\hline Cough (without fever) & 4 & 8.0 & 37 & 3.2 & 3.8 & $1.2-11.5$ & 0.019 \\
\hline Sore throat (without fever) & 0 & 0 & $20 / 1,172$ & 1.7 & 0.7 & $0.04-11.7$ & $0.8^{\mathrm{c}}$ \\
\hline Rhinitis (without fever) & 0 & 0 & 8 & 0.7 & 1.6 & $0.08-27.8$ & $0.8^{c}$ \\
\hline Dyspnoea (without fever) & 3 & 6.0 & $44 / 1,170$ & 3.8 & 2.4 & $0.7-8.7$ & 0.17 \\
\hline Fatigue & 3 & 6.0 & $37 / 1,171$ & 3.2 & 3.1 & $0.9-11.2$ & 0.077 \\
\hline Diarrhoea & 3 & 6.0 & $26 / 1,171$ & 2.2 & $4 \cdot 3$ & $1.2-15.4$ & 0.026 \\
\hline Myalgia & 2 & 4.0 & 3 & 0.3 & 22.3 & $4.01-123.7$ & $<0.001^{c}$ \\
\hline Headache & 0 & 0 & $18 / 1,172$ & 1.5 & 0.6 & $0.03-10.6$ & 0.7 \\
\hline Vomiting & 1 & 2.0 & 37 & 3.2 & 0.9 & $0.1-7.1$ & 0.94 \\
\hline Dysgeusia/dysosmia & 2 & 4.0 & 1 & 0.09 & 28.9 & $3 \cdot 7-224.8$ & $0.001^{\mathrm{c}}$ \\
\hline At least one symptom ${ }^{d}$ & 19 & 38.0 & 263 & 22.4 & $3 \cdot 3$ & $(1.8-6.3)$ & $<0.001$ \\
\hline \multicolumn{8}{|l|}{ Clinical findings } \\
\hline \multirow{2}{*}{ Temperature, median ${ }^{\circ} \mathrm{C}(\mathrm{IQR})$} & \multicolumn{2}{|c|}{$\mathrm{n}=20$} & \multicolumn{2}{|c|}{$n=652$} & \multirow{2}{*}{1.3} & \multirow{2}{*}{$(0.7-2.3)$} & \multirow{2}{*}{0.4} \\
\hline & 36.8 & $-37 \cdot 5)$ & 36.9( & 37.3) & & & \\
\hline \multirow{2}{*}{ O2 saturation, \% (IQR) } & \multicolumn{2}{|c|}{$\mathrm{n}=19$} & \multirow{2}{*}{\multicolumn{2}{|c|}{$n=632$}} & \multirow{2}{*}{0.9} & \multirow{2}{*}{$(0.9-1.0)$} & 8 \\
\hline & & 98) & & & & & 0.08 \\
\hline
\end{tabular}

$\mathrm{Cl}$ : confidence interval; IQR: interquartile range; SARS-CoV-2: severe acute respiratory syndrome coronavirus 2.

a Number of observations is $n$ if not otherwise specified.

${ }^{b}$ Adjusted for sex and age.

c Penalised maximum likelihood logistic regression (Firth model).

d The individual had at least one COVID-19-related symptom, irrespective of which symptom (fever, cough, dyspnoea, nausea, vomiting, diarrhoea, myalgia, headache, fatigue) before the testing procedure.

Adults are considered those aged $\geq 18$ years. Data on children (aged $<18$ years) are presented in Supplementary File S3. 
As a secondary analysis, the weekly positivity rate in patients without high-risk symptoms, e.g. fever and dyspnoea, was estimated over the entire study period and compared with patients admitted to the hospital with high clinical suspicion and also with the cumulative COVID-19 hospitalisation rate in Region Stockholm using Spearman's rank correlation coefficient. The hospitalisation rate was preferred to the incidence at the community level since it reflects better the evolution of the epidemic in the region because of the limited testing capacity outside of hospitals.

Data on sociodemographic level of municipalities were extracted from the Statistical agency of Sweden [16].

Data for the COVID-19 weekly Stockholm hospitalisation rate were facilitated by the Centrum for Epidemiology and Community Medicine (https://ces.sll.se).

\section{Statistical methods}

Summary statistics were presented as proportions for categorical variables and as means with standard deviations (SD) for normally distributed continuous variables or medians with interquartile range (IQR) for skewed continuous variables. Associations were examined with a t-test for normally distributed continuous variables, a Wilcoxon-Mann-Whitney $U$ test for variables not following a normal distribution and a chi-squared test or Fisher's exact test for categorical variables, as appropriate. The overall positivity rate of SARS-CoV-2 infection was described using 95\% confidence intervals $(\mathrm{Cl})$. Positivity rate estimates were also obtained for the 4 -week period when information on screening or reason for testing was available from the medical records. Outcomes of statistical tests were considered significant when two-sided $p<0.05$. A multivariable logistic regression with penalised maximum likelihood estimation was used to identify associations between patient characteristics and a positive test. Variables that were assessed as clinically relevant or those with a significance level of $p<0.1$ in the univariate analysis were included for multivariable analysis. Data analyses were performed using STATA (version 16, STATACorp, College Station, Texas, United States).

The study has been reported following the Strengthening the Reporting of Observational Studies in Epidemiology (STROBE) guidelines for reporting observational studies.

\section{Ethical statement}

Ethical approval to use the research database was obtained from the Regional Ethical Review Board in Stockholm (Dnr-2018/1030-31, amendment 202001385). The medical chart review was performed as part of the quality control and safety tasks of the hospitals.

\section{Results}

In total, 36,249 in-hospital healthcare episodes were reported from 16 March to 27 September 2020 and, of these, 24,245 (66.9\%) were tested for
SARS-CoV-2 by PCR at admission ( $98.5 \%$ nasopharyngeal swabs and $1.5 \%$ throat swabs). Among these, $38.8 \%(9,415 / 24,245)$ of SARS-CoV-2-tested healthcare episodes presented with high clinical suspicion, whereas $61.2 \%(14,830 / 24,245)$ of SARS-CoV-2-tested healthcare episodes included patients that presented with low clinical suspicion upon hospital admission. Of these healthcare episodes, 3.2\% $(469 / 14,830)$ were positive for SARS-CoV-2 (Figure 1).

Between 16 March to 12 April 2020, the period targeted for medical chart review, there were 5,369 healthcare episodes in 5,091 individuals; SARS-CoV-2 tests were conducted in $55.2 \%(2,963 / 5,369)$ of healthcare episodes, and $57.0 \%(2,901 / 5,091)$ of these patients. After exclusion of patients transferred from other hospitals $(n=138)$, patients with a high COVID-19 suspicion $(n=1,303)$, and healthcare episodes without information about the symptoms $(n=25), 1,497$ episodes (from 1,483 patients) were tested and defined as belonging to care episodes with low COVID-19 suspicion (Figure 1).

In total, $39.2 \%(259 / 661)$ of healthcare episodes in children less than 18 years of age were tested with low COVID-19 suspicion, compared with $38.9 \%(1,237 / 3,183)$ of healthcare episodes in adults $(p=0.9 ; n=59$ missing values on age, of which one was positive and 58 were negative). The testing rate varied across hospital units, which was higher in the maternity units $(63.7 \%$; $411 / 645)$ compared with the adult surgical or medical emergency units $(42.1 \% ; 215 / 511)$, paediatric units (38.9\%; 231/594), surgical units $(38.0 \% ; 222 / 585)$, and the medical units $(24.4 \% ; 291 / 1,195 ; p<0.001)$. On 25 March, several units in the hospital implemented routine SARS-CoV-2 testing at admission. The COVID19 low suspicion testing rate was $19.0 \%(322 / 1,694)$ before 25 March, compared with $53.2 \%(1,175 / 2,209)$ after introducing routine testing ( $p<0.001)$. In the maternity units, the testing rate changed from $11.9 \%$ (28/235) before 25 March to 93.4\% (383/410) afterwards, and was the unit that had the greatest increase in routine testing rate $(p<0.001)$.

When the reason for testing was investigated among the 1,497 healthcare episodes tested for SARS-CoV-2 with low suspicion, $21.9 \%(328 / 1,497)$ of the episodes were tested before delivery, $21.7 \%$ were $(325 / 1,497)$ before surgery or other invasive procedure, and $56.4 \%$ $(844 / 1,497)$ were because of any other reason for hospital admission.

When comparing the cumulative COVID-19 hospitalisation rate in Region Stockholm per week with the SARS-CoV-2 positivity rate in patients without fever or dyspnoea admitted from March 25 (implementation of more extensive testing at the hospital) until September 27 , there was a strong correlation (rho=0.93; $p<0.001$, Spearman's rank correlation coefficient) (Figure $2 \mathrm{~A}$ and $2 \mathrm{~B}$ ). 


\section{TABLE 3}

Multivariable logistic regression model of risk factors for SARS-CoV-2 infection in patients with low COVID-19 suspicion, Karolinska University Hospital, Stockholm, Sweden, 16 March-12 April $2020(\mathrm{n}=1,482)$

\begin{tabular}{|c|c|c|c|}
\hline Characteristics & $\mathrm{aOR}$ & $95 \% \mathrm{Cl}$ & $\mathrm{p}$ value \\
\hline Age & 1.0 & $0.99-1.02$ & 0.7 \\
\hline Sex (male) & 0.5 & $0.3-1.1$ & 0.09 \\
\hline \multicolumn{4}{|l|}{ Reason for being tested } \\
\hline Hospital admission & Reference & NA & NA \\
\hline Before surgery & 1.2 & $0.5-2.8$ & 0.6 \\
\hline Before delivery & 2.6 & $1.3-5.4$ & 0.008 \\
\hline \multicolumn{4}{|l|}{ Comorbidities and symptoms } \\
\hline Cancer & 0.5 & $0.2-1.4$ & 0.2 \\
\hline $\begin{array}{l}\text { Fever (without respiratory } \\
\text { symptoms) }\end{array}$ & 2.9 & $1.2-7.4$ & 0.02 \\
\hline Cough (without fever) & 4.0 & $1.4-12.0$ & 0.012 \\
\hline Diarrhoea & 3.7 & $1.1-12.3$ & 0.03 \\
\hline Myalgia & $14 \cdot 5$ & $2.6-81.2$ & 0.002 \\
\hline
\end{tabular}

$\mathrm{Cl}$ : confidence interval; COVID-19: coronavirus disease; OR: odds ratio; SARS-CoV-2: severe acute respiratory syndrome coronavirus 2.

Reference category for binary variables (cancer, fever, cough, diarrhoea, and myalgia) was not having any of these conditions. Having dysgeusia/dysosmia was not included in the final model since the number of events were very small. Penalised maximum likelihood logistic regression model adjusted by sex, age, reason for being tested, comorbidity of cancer and having fever, cough, diarrhoea, and myalgia.

In analyses restricted to maternity wards, the overall positivity rate was $3.7 \%(149 / 4,031)$ from 25 March until 27 September, and the positivity rate was also correlated with the Region Stockholm cumulative COVID-19 hospitalisation rate over time (rho $=0.75$; p<0.001, Spearman's rank correlation coefficient) (Supplementary File S2).

When comparing the weekly SARS-CoV-2 positivity rate in patients admitted with and without fever or dyspnoea at Karolinska University Hospital, there was also a strong correlation with positivity rates in those tested with high or low clinical suspicion of COVID-19 (rho $=0.92 ; p<0.001$, Spearman's rank correlation coefficient) (Figure $2 \mathrm{C}$ ).

\section{Factors associated with SARS-CoV-2 positivity} rate among patients with a low clinical COVID-19 suspicion

The main characteristics of the 1,482 patients (one patient with missing age was excluded from the analysis) with the first healthcare episodes used for each patient tested with low suspicion of COVID-19 are presented by reason for being tested and by age group (adults vs children) (Table 1 ).

Among the 1,482 patients with low COVID-19 suspicion from 16 March to 12 April, the SARS-CoV-2 positivity rate was $3.8 \%(57 / 1,482)$, and was $6.0 \%(22 / 368)$ in symptomatic patients compared with $3.1 \%(35 / 1,114$; $\mathrm{p}=0.014$ ) in asymptomatic patients. The positivity rate in patients with low COVID-19 suspicion varied across the weeks from $1.7 \%(2 / 117)$ in the third week of March to $4.5 \%(22 / 488)$ in the first week of April. In patients with low COVID-19 suspicion, women had a higher positivity rate $(5.0 \% ; 45 / 893)$ compared with men $(2.0 \%$; $12 / 589 ; p<0.003)$, but when we exclude the group of pregnant women tested before delivery from the analysis, there was no difference in the positivity rate by $\operatorname{sex}(3.7 \% ; 21 / 566$ vs $2.0 \% ; 12 / 589 ; p=0.13)$. Adults had a SARS-CoV-2 positivity rate of $4.1 \%(50 / 1,223)$, and children of $2.7 \%(7 / 259 ; p=0.3)$, with a median age among SARS-CoV-2-positive adults of 49.4 years (IQR: 33.0-68.3) and 5.6 years (IQR: 0.4-12.3) among SARS-CoV-2-positive children. The age group 18-45 years had a higher positivity rate $(5.6 \% ; 32 / 570)$ compared with children $(2.7 \% ; 7 / 259)$, and compared with adults over 45 years of age $(2.8 \% ; 18 / 653 ; p=0.02)$, although there was no significant difference with the group aged $18-45$ years if the pregnant women tested before delivery were excluded (3.3\%; 8/243; $p=0.9)$.

The positivity rate among patients with low clinical suspicion of COVID-19 was highest among women before delivery $(7.3 \% ; 24 / 327)$, followed by those admitted to the hospital for reasons other than surgery or delivery $(3.0 \% ; 25 / 831)$, and before surgery or other invasive procedure $(2.5 \% ; 8 / 324)$ (Table 1 ). Among asymptomatic patients, the equivalent rates were $6.2 \%$ (20/322), $1.9 \%(10 / 517)$ and $1.8 \%(5 / 275)$ for those groups, respectively $(p=0.001)$.

Characteristics associated with a SARS-CoV-2 infection in patients with low clinical suspicion of COVID-19

Because of the low number of SARS-CoV-2 positive patients among children with low suspicion $(n=7$; $2.7 \%$ ), separate analyses were performed, stratified for adults and children; analysis with the data for children are presented in Supplementary File S3.

In the adult study population, male sex was associated with a lower PCR positivity rate of SARS-CoV-2 after adjusting for age (odds ratio (OR): $0.4 ; 95 \% \mathrm{Cl}: 0.2-$ $0.8 ; p=0.02$ ). Having a neoplasia was negatively but not significantly associated with SARS-CoV-2 positivity (8\%; 4/50 vs 22.1\%; 259/1,171 (OR: $0.4 ; 95 \% \mathrm{Cl}: 0.1-$ 1.2; $p=0.09)$ ), after adjusting by age and sex. In total, $38.0 \%(19 / 50)$ patients with a positive SARS-CoV-2 PCR test had at least one symptom associated with COVID19 compared with $263 / 1,173(22.4 \%)$ for patients with a negative test (OR: 3.3; 95\% Cl: 1.8-6.3; $\mathrm{p}<0.001$ ), despite being regarded as having low suspicion for COVID-19 according to the medical notes. Five out of $50(10.0 \%)$ individuals with a positive SARS-CoV-2 test were reporting fever without any other respiratory symptoms (OR: $2.8 ; 95 \% \mathrm{Cl}: 1.1-7.6 ; \mathrm{p}=0.039)$. In addition, $8 \%$ (4/50) were reporting cough without fever associated (OR: 3.8; 95\% Cl: 1.2-11.5; $\mathrm{p}=0.019$ ). Furthermore, diarrhoea $(3 / 50 ; 6.0 \%)$ and myalgia $(2 / 50 ; 4.0 \%)$ were also associated with SARS-CoV-2 
positivity after adjusting for age and sex (OR: 4.3; 95\% Cl: 1.2-15.4; $p=0.026$ and OR: 22.3; $95 \% \mathrm{Cl}$ : 4.01-123.7; $p<0.001$, respectively) (Table 2). A sensitivity analysis was performed restricting the analysis to those tests conducted at hospital admission after the implementation of the routine testing recommendation at hospital admission (25 March) without any substantial changes in the associations found (Supplementary File S4). $^{2}$

Fever and diarrhoea were the only symptoms significantly associated with SARS-CoV-2 positivity in children after adjusting for age and sex (Supplementary File S4).

Using a penalised maximum likelihood multivariable logistic regression model that included age, sex, reason for testing and clinical symptoms, the factors associated with SARS-CoV-2 positivity were being tested before delivery (adjusted OR (aOR): 2.6; 95\% Cl: 1.3-5.4), and having isolated symptoms such as fever (aOR: $2.9 ; 95 \% \mathrm{Cl} 1.2-7.4$ ), cough (aOR $4.0 ; 95 \% \mathrm{Cl}$ : 1.4-12.0), diarrhoea (aOR: 3.7; 95\% Cl: 1.1-12.3) and myalgia (aOR: $14.5 ; 95 \% \mathrm{Cl} 2.6-81.2$ ) (Table 3 ).

\section{Discussion}

Our study reveals a SARS-CoV-2 test positivity rate of almost $4 \%$ among patients with low COVID-19 suspicion at hospital admission during a 4-week period (16 March-12 April) in 2020 when SARS-CoV-2 community transmission was widely established in Region Stockholm [15]. During this period, around $75 \%$ $(1,115 / 1,482)$ of the patients tested with low suspicion were asymptomatic for COVID-19 symptoms, whereas $25 \%(368 / 1,482)$ of patients reported some isolated symptoms, which could be associated with COVID-19 but did not trigger an obvious COVID-19 suspicion by health professionals. In addition, we have excluded those patients who were tested because of a high COVID-19 suspicion; this group had a positivity rate around $40 \%$ during the 4 -week period (data not shown).

Although patients defined in our study as having low COVID-19 suspicion might not be exactly comparable to a systematically screened hospital population, they represent a patient group in which the decision of performing a SARS-CoV-2 test should be taken at admission to the hospital. The two patient populations in our hospital who were screened more systematically, i.e. before surgery or other invasive procedures in adults and before delivery in pregnant women, had a positivity rate of $2.7 \%$ and $7.3 \%$, respectively.

The SARS-CoV-2 test positivity rate we observed in asymptomatic patients ( $>3 \%$ ) was similar to that reported in another study during their peak of the epidemic during the first pandemic wave [17], although our estimate is higher compared with screening studies reporting hospital positivity rates below 1\% which were conducted in areas with low incidence rates $[18,19]$. In our study, four of every 100 patients with low COVID-19 suspicion ( $3 / 100$ in asymptomatic patients) tested positive for SARS-CoV-2 with PCR; this suggests that, in a universal SARS-CoV-2 hospital testing scenario with an established community transmission, the numbers needed to test (NNT) to detect one positive patient with low COVID-19 suspicion would be 26 .

The positivity rate in low COVID-19 suspicion cases correlated fairly well with the positivity rate in those with a high COVID-19 suspicion in our study, as well as with the hospitalisation rate in Region Stockholm. In this regard, if the accepted NNT to detect one positive patient in low suspicion patients (a proxy for screening) is 100 , our data indicates that this would occur when the positivity rate among high-risk patients exceeds $1.8 \%$. If the accepted NNT is 50 , the equivalent positivity rate among high-risk patients is $3.8 \%$. The corresponding figures based on the cumulative COVID-19 hospitalisation rate per week were 1.8 and 3.9/100,000 population. Therefore, the positivity rate of patients with COVID-19 symptoms and the cumulative COVID19 hospitalisation rate per week could be used to estimate the transmission risk at the hospital. In this regard, and as per recommended by ECDC, the testing approach at hospital admission needs to consider the epidemiological situation in the community served by the hospital. Thus, if the SARS-CoV-2 transmission in the community served by the hospital is very low or absent, universal testing should not be implemented [12]. Additional studies should be designed to elucidate and validate the cut-off value observed in our study, to further guide hospital screening implementation.

The overall positivity rate of women was higher compared with men, although there was no difference when pregnant women admitted before delivery were excluded. In this regard, when comparing the reasons for SARS-CoV-2 testing among those with low suspicion, the SARS-CoV-2 test positivity rate in pregnant women presenting for delivery was undoubtedly higher compared with other screening purposes such as hospital admission or testing before surgery or any other invasive procedure. This was particularly manifested in asymptomatic patients, with a positivity rate over $6 \%$ observed in asymptomatic pregnant women before delivery. This high positivity rate has also been reported in other studies with ranges varying from 1.4 to $14.5 \%$, depending on the community prevalence of the area [18,20-22]. On one hand, a plausible explanation is that based on the association of COVID-19 with preeclampsia [23]. On the other hand, most pregnant women with preeclampsia may have been referred to the Karolinska University hospital, where the COVID-19 diagnosis was performed, capturing more COVID-19positive pregnant women and therefore overestimating the positivity rate found in our study.

Among patients with low suspicion of COVID-19, cancer was associated with a lower SARS-CoV-2 positivity rate, around $0.5 \%$ in asymptomatic patients. This low rate suggests that patients with this condition may have 
adjusted their behaviour, e.g. isolated themselves, and avoided exposure to SARS-CoV-2. However, despite the fact that the SARS-CoV-2 prevalence rate in oncological patients is lower compared with patients in other units such as maternity wards [18], the baseline testing for patients with cancer has been undoubtedly proposed in all inpatients admitted to oncology/haematology units and before starting immunosuppressive chemotherapy [24].

Therefore, when implementing a universal screening policy at hospital admission, testing women presenting for delivery should be a core strategy to reduce the risk of SARS-CoV-2 transmission in healthcare facilities [25], particularly since only around 14 pregnant women were needed to test before delivery to detect 1 positive case. However, concerning testing before elective surgery and other invasive procedures or before hospital admission, we report a lower positivity rate, resulting respectively in 40 and 32 NNT to identify one positive SARS-CoV-2 case, which is slightly higher in asymptomatic patients. Thereafter, for the evaluation of the cost-effectiveness of universal screening policies at hospital admission, other aspects should be considered $[19,26]$ such as the community incidence of infection, since an escalating COVID-19 incidence may increase potential benefits of this policy. In a study from Japan in an area with low levels of community SARS-CoV-2 transmission, the positivity rate was $0.03 \%$ (only two cases among more than 6,000 patients tested). This suggests that a universal SARSCoV-2 testing strategy might be labour-intensive and not cost-effective in areas with low infection rates [26]. Also, the strategy will depend on the variations of the community transmission level across regions or municipalities [27] and also over time. We show that the positivity rate remained high the first months of the epidemic and subsequently declined in the following months, although the testing rate remained stable. In this regard, we propose the use of the positivity rate of patients with COVID-19 symptoms who will definitely be tested for estimating the transmission risk at the hospital in asymptomatic patients and to guide the implementation of screening testing. In addition, other potential benefits to consider are the prevention of unnecessary risks of surgery performed on someone with an underlying COVID-19 infection [28].

There are also barriers and challenges to the implementation of standardised COVID-19 screening programmes. These include the diagnostic testing capacity of the health services, logistical issues affecting sampling and turnaround times [5], and also the possibility of false-negative results for patients tested during the incubation period, for which a re-testing procedure after 5-7 days has been proposed [29].

We identified clinical predictors for a positive SARSCoV-2 test in patients with low COVID-19 suspicion. In the multivariable model, isolated symptoms, e.g. selfreported fever (without cough), cough (without fever), diarrhoea and myalgia, were associated with test positivity after adjustment for age, sex, the reason for testing and other clinical parameters. These findings indicate that vigilance for possible SARS-CoV-2 infection should remain high, even in patients with primary suspicion of other diagnosis when there is any symptom indicative of SARS-CoV-2, at least when community spread is high.

The low number of SARS-CoV-2-positive children precluded us from performing separate multivariate analyses in children. However, univariate results are in line with other studies where the symptom-based testing strategy failed to detect up to $45 \%$ of children [30] suggesting that a symptom-based testing strategy could lead to a substantial increased risk of intra-hospital transmission.

A strength of the study is that we included complete data from one hospital during a period of almost 7 months with more than 24,000 healthcare episodes reviewed and with an in-depth manual record review of almost 3,000 healthcare episodes. A limitation of the study is the retrospective design based on clinical routine data, and in some cases, the reasons for testing may have been poorly registered by health professionals while the reason for testing with low COVID19 suspicion may be overestimated. To reduce the risk of misclassification, we excluded patients tested because of high COVID-19 suspicion and patients with more than one symptom suggestive of COVID-19. Our study has limited generalisability to areas with different rates of SARS-CoV-2 infections. Furthermore, we cannot conclude that our findings are representative of all patients diagnosed with COVID-19 since the study only included patients admitted to the hospital.

\section{Conclusions}

This study reports a high SARS-CoV-2 test positivity rate in patients with low COVID-19 suspicion at hospital admission in Stockholm from March to September 2020. Universal testing before delivery and testing in those patients with any isolated symptom despite having low COVID-19 suspicion should be considered among SARS-CoV-2 testing strategies at the hospital level, although the background incidence at the community should be considered. Positivity rates in hospitalised patients with high-suspicion of COVID-19 can guide SARS-CoV-2 screening implementation.

\section{COVID-19-data-review collaborators:}

Isabelle Johansson, Victoria Hovergren, David Björklund, Allan Zhao, Oscar Backrud, Jesper Ahlberg, Emilie Homlkvist, Johanna Lundquist

\section{Acknowledgements}

We would like to acknowledge to Marie-Christine Berglund from IT department of Karolinska University Hospital who helped us to extract data from the health information system of the Karolinska University Hospital, and also Maria-Pia 
Hergens who provided us with the data about the COVID-19 hospitalisation rate in Region Stockholm.

Funding: Ana Requena-Mendez is funded from Strategic Research Program in Epidemiology at Karolinska Institutet. This study has been partially funded by the Swedish Innovation Agency (Vinnova- 2016-00563), Emil and Wera Cornells Stiftelse, and Region Stockholm.

\section{Conflict of interest}

None declared.

\section{Authors' contributions}

PN and AM did the study design, coordinated the manual annotation of the medical records, collaborated in the data analysis and reviewed and supervised the manuscript. $\mathrm{PH}$, SvdW, HT coordinated the data extraction, collaborated in the data analysis, and reviewed the manuscript. $\mathrm{OH}, \mathrm{AF}$ and FN collaborated in the study design, supervised the data extraction and data analysis and reviewed the manuscript. ARM collaborated in the study design and data extraction, coordinated and performed the data analysis and drafted the manuscript. The COVID-19-data-review collaborators performed the manual annotation of the medical records.

\section{References}

1. Dong E, Du H, Gardner L. An interactive web-based dashboard to track COVID-19 in real time. Lancet Infect Dis. 2020;20(5):533-4. https://doi.org/10.1016/S14733099(20)30120-1 PMID: 32087114

2. World Health Organization (WHO). COVID-19 Strategy Update. Geneva: WHO; 2020. Available from: https://www.who.int/ docs/default-source/coronaviruse/covid-strategy-update14april2020.pdf

3. Cheemarla NR, Brito AF, Fauver JR, Alpert T, Vogels CBF, Omer SB, et al. Host response-based screening to identify undiagnosed cases of COVID-19 and expand testing capacity. medRxiv2020.06.04.20109306. Preprint. https://doi.org/10.11 01/2020.06.04.20109306

4. Benítez MA, Velasco C, Sequeira AR, Henríquez J, Menezes FM, Paolucci F. Responses to COVID-19 in five Latin American countries. Health Policy Technol. 2020;9(4):525-59. https:// doi.org/10.1016/j.hlpt.2020.08.014 PMID: 32874863

5. Rivett L, Sridhar S, Sparkes D, Routledge M, Jones NK, Forrest $\mathrm{S}$, et al. Screening of healthcare workers for SARS-CoV-2 highlights the role of asymptomatic carriage in COVID-19 transmission. eLife. 2020;9:e58728. https://doi.org/10.7554/ eLife.58728 PMID: 32392129

6. Ra SH, Lim JS, Kim GU, Kim MJ, Jung J, Kim S-H. Upper respiratory viral load in asymptomatic individuals and mildly symptomatic patients with SARS-CoV-2 infection. Thorax. 2021;76(1):61-3. https://doi.org/10.1136/ thoraxjnl-2020-215042 PMID: 32963115

7. Ravindra K, Malik VS, Padhi BK, Goel S, Gupta M. Asymptomatic infection and transmission of COVID-19 among clusters: systematic review and meta-analysis. Public Health. 2022;203:100-9. https://doi.org/10.1016/j.puhe.2021.12.003 PMID: 35038628

8. Porcel-Vazquez JA, Andres-Peiro JV, Garcia-Sanchez Y, GuerraFarfan E, Mestre-Torres J, Minguell-Monyart J, et al. Usefulness of PCR screening in the initial triage of trauma patients during COVID-19 pandemic. J Orthop Trauma. 2020;34(10):e377-81. https://doi.org/10.1097/BOT.0000000000001903 PMID: 32947588

9. Goldfarb IT, Diouf K, Barth WH Jr, Robinson JN, Katz D, Gregory $\mathrm{KE}$, et al. Universal SARS-CoV-2 testing on admission to the labor and delivery unit: Low prevalence among asymptomatic obstetric patients. Infect Control Hosp Epidemiol. 2020;41(9):1095-6. https://doi.org/10.1017/ice.2020.255 PMID: 32456729

10. Black JRM, Bailey C, Przewrocka J, Dijkstra KK, Swanton C. COVID-19: the case for health-care worker screening to prevent hospital transmission. Lancet. 2020;395(10234):1418-20.
https://doi.org/10.1016/S0140-6736(20)30917-X PMID: 32305073

11. Story D, Coyle E, Devapalasundaram A, Sidiropoulos S, Ou Yang B, Coulson T. Documenting COVID-19 screening before surgery during lockdown (COVID Screen): an audit with routinely collected health data. Aust Health Rev. 2020;44(5):723-7. https://doi.org/10.1071/AH20169 PMID: 32962798

12. European Centre for Disease Prevention and Control (ECDC). COVID-19 testing strategies and objectives. Stockholm: ECDC. [Accessed: 28 Jan 2022]. Available from: https://www.ecdc.europa.eu/en/publications-data/ covid-19-testing-strategies-and-objectives

13. Marshall S, Ishikawa M, Tanaka H, Sakura H, Uchigata Y. Universal testing for SARS-CoV-2 infection on admission among hospitalized emergency patients: A survey report from a single facility in Tokyo. Infect Dis Health. 2022;27(1):58-60. https://doi.org/10.1016/j.idh.2021.09.002 PMID: 34627745

14. World Health Organization (WHO). WHO Coronavirus Disease (COVID-19) Dashboard. Geneva: WHO. [Accessed: 15 Nov 2020]. Available from: https://covid19.who.int/region/euro/country/se

15. Folkhälsomyndigheten (FOHM). Antal fall av Covid-19 i Sverige på regionnivå. [Number of cases of COVID-19 in Sweden at the regional level]. Stockholm: FOHM. [Accessed: 16 Nov 2020]. Swedish. Available from: https://fohm.maps.arcgis.com/apps/ opsdashboard/index.html\#/21a20798598b4badb417ff63dbc9 $63 \mathrm{dc}$

16. Statistiska centralbyrån (SCB). Förmögenhetsstatistik för personer efter region, kön, ålder, tillgångar/skulder. År $2004-2007$. [Wealth statistics for persons by region, sex, age, assets / liabilities. Year 2004 - 2007l. Stockholm: SCB. [Accessed: 6 Dec 2020]. Swedish. Available from: http://www.statistikdatabasen.scb.se/pxweb/sv/ssd/ START__HE__HE0104/TillgOversiktAldReg

17. Aslam A, Singh J, Robilotti E, Chow K, Bist T, Reidy-Lagunes $D$, et al. Severe acute respiratory syndrome coronavirus 2 surveillance and exposure in the perioperative setting with universal testing and personal protective equipment policies. Clin Infect Dis. 2021;73(9):e3013-8. https://doi.org/10.1093/ cid/ciaa1607 PMID: 33090210

18. Shah MA, Mayer S, Emlen F, Sholle E, Christos P, Cushing $M$, et al. Clinical screening for COVID-19 in asymptomatic patients with cancer. JAMA Netw Open. 2020;3(9):e2023121. https://doi.org/10.1001/jamanetworkopen.2020.23121 PMID: 32990737

19. Sastry SR, Pryor R, Raybould JE, Reznicek J, Cooper K, Patrick $A$, et al. Universal screening for the SARS-CoV-2 virus on hospital admission in an area with low COVID-19 prevalence. Infect Control Hosp Epidemiol. 2020;41(10):1231-3. https://doi. org/10.1017/ice.2020.358 PMID: 32698924

20. Sutton D, Fuchs K, D’Alton M, Goffman D. Universal screening for SARS-CoV-2 in women admitted for delivery. N Engl J Med. 2020;382(22):2163-4. https://doi.org/10.1056/NEJMc2009316 PMID: 32283004

21. Yassa M, Yirmibes C, Cavusoglu G, Eksi H, Dogu C, Usta C, et al. Outcomes of universal SARS-CoV-2 testing program in pregnant women admitted to hospital and the adjuvant role of lung ultrasound in screening: a prospective cohort study. Matern Fetal Neonatal Med. 2020;33(22):3820-6. https://doi. org/10.1080/14767058.2020.1798398 PMID: 32691641

22. Tanacan A, Erol SA, Turgay B, Anuk AT, Secen EI, Yegin GF, et al. The rate of SARS-CoV-2 positivity in asymptomatic pregnant women admitted to hospital for delivery: Experience of a pandemic center in Turkey. Eur J Obstet Gynecol Reprod Biol. 2020;253:31-4. https://doi.org/10.1016/j.ejogrb.2020.07.051 PMID: 32763728

23. Leavitt AO, Li Q, Chan ED. Re: Pre-eclampsia-like syndrome induced by severe COVID-19: a prospective observational study: Common pathophysiology of pre-eclampsia and severe COVID-19? BJOG. 2021;128(3):618-9. https://doi. org/10.1111/1471-0528.16584 PMID: 33225566

24. Ontario Ministry of Health. COVID-19 Provincial Testing Guidance Update. Ontario: Ministry of Health. [Accessed: 29 Jan 2022]. Available from: https://www.rhra.ca/wp-content/ uploads/2021/05/2021 testing guidance.pdf

25. Díaz-Corvillón P, Mönckeberg M, Barros A, Illanes SE, Soldati A, Nien JK, et al. Routine screening for SARS CoV2 in unselected pregnant women at delivery. PLoS One. 2020;15(9):e0239887. https://doi.org/10.1371/journal. pone.0239887 PMID: 32991621

26. Nakamura I, Itoi T. Universal PCR screening for coronavirus disease 2019 in asymptomatic patients on admission. Clin Microbiol Infect. 2021;27(4):658-9. https://doi.org/10.1016/j. cmi.2020.11.010 PMID: 33221431

27. Lundkvist $\AA$, Hanson S, Olsen B. Pronounced difference in Covid-19 antibody prevalence indicates cluster 
transmission in Stockholm, Sweden. Infect Ecol Epidemiol.

2020;10(1):1806505. https://doi.org/10.1080/20008686.2020. 1806505 PMID: 32944166

28. Kahlberg A, Mascia D, Bellosta R, Attisani L, Pegorer

$M$, Socrate AM, et al. Vascular surgery during COVID-19

emergency in hub hospitals of Lombardy: Experience on 305

patients. Eur J Vasc Endovasc Surg. 2021;61(2):306-15. https:// doi.org/10.1016/j.ejvs.2020.10.025 PMID: 33262093

29. National Health Service (NHS). Healthcare associated COVID-19 infections - further action. London: NHS; 2020. Available from: https://www.england.nhs.uk/coronavirus/wp-content/ uploads/sites/52/2020/06/Healthcare-associated-COVID-19infections--further-action-24-June-2020.pdf

30. Poline J, Gaschignard J, Leblanc C, Madhi F, Foucaud E, Nattes E, et al. Systematic Severe acute respiratory syndrome coronavirus 2 screening at hospital admission in children: a French prospective multicenter study. Clin Infect Dis. 2021;72(12):2215-7. https://doi.org/10.1093/cid/ciaa1044 PMID: 32710743

\section{License, supplementary material and copyright}

This is an open-access article distributed under the terms of the Creative Commons Attribution (CC BY 4.0) Licence. You may share and adapt the material, but must give appropriate credit to the source, provide a link to the licence and indicate if changes were made.

Any supplementary material referenced in the article can be found in the online version.

This article is copyright of the authors or their affiliated institutions, 2022. 